\title{
Kollaboratives Arbeiten mit Robotern - Vision und realistische Perspektive
}

\author{
Michael Haag
}

Während in Deutschland der Begriff „Industrie 4.0“ in aller Munde ist, proklamieren Erik Brynjolfsson und Andrew McAfee, zwei Wissenschaftler am Center for Digital Business am Massachusetts Institute of Technology (MIT), das „,zweite Maschinenzeitalter“ (The Second Machine Age) (Pearlstein 2014; Braunberger 2014). Demnach steigen die Rechenleistung, die Datenmenge und die Anzahl der Sensoren in unserer Welt derart, dass Rechner künftig in der Lage sind, (Gedanken-)Leistungen zu erbringen, die bislang dem Menschen vorbehalten sind und eher im Reich des Science-Fiction anzusiedeln waren. Was für die Verbraucher gut ist, da diese sehr individualisierte Produkte zu erschwinglichen Preisen erwarten können, wird gleichzeitig die Arbeits- und Wirtschaftswelt drastisch verändern. Als Beispiel nennen Brynjolfsson und McAfee die Internet-Plattform Instagram, die es Benutzern erlaubt, Fotos und Videos zu erstellen und mit anderen Nutzern zu teilen. Als das Unternehmen von Facebook für $1 \mathrm{Mrd}$. US\$ übernommen wurde, beschäftigte es gerade einmal zwölf Mitarbeiter, während der traditionelle Foto-Hersteller Kodak in seinen besten Zeiten 150.000 Mitarbeiter zählte. Der wirtschaftliche Erfolg eines Unternehmens schlägt sich im digitalen Zeitalter somit nicht mehr automatisch auf gut bezahlte Arbeitsplätze nieder. Nach Brynjolfsson und McAfee wird es zwei Gruppen von Beschäftigten geben: diejenigen, die den Computern sagen, was sie tun sollen, und diejenigen, die umgekehrt Anweisungen von Computern befolgen.

Auf die industrielle Produktion bezogen, in der nicht nur Bits und Bytes virtuell hinund hergeschoben werden, sondern Güter physisch bewegt und Produkte hergestellt werden, stellt sich die Frage, wie sich die Arbeitswelt für die Menschen verändern wird. Zwar werden Serienproduktionen zunehmend automatisiert, Maschinen und flexible Roboter sind auf dem Vormarsch. Dennoch waren nach den Erhebungen des Statistischen Bundesamtes in den Betrieben des Verarbeitenden Gewerbes mit 50 und mehr Beschäf-

Der Originaltext dieses Beitrags wurde überarbeitet. Das vollständige Korrekturverzeichnis finden Sie am Ende des Buchs und online unter http://dx.doi.org/10.1007/978-3-662-45915-7_17.

M. Haag ( $\varangle$ )

KUKA Roboter GmbH, Zugspitzstr. 140, 86165 Augsburg, Germany

e-mail: MichaelHaag@Kuka-roboter.de

A. Botthof, E.A. Hartmann (Hrsg.), Zukunft der Arbeit in Industrie 4.0,

DOI 10.1007/978-3-662-45915-7_6 


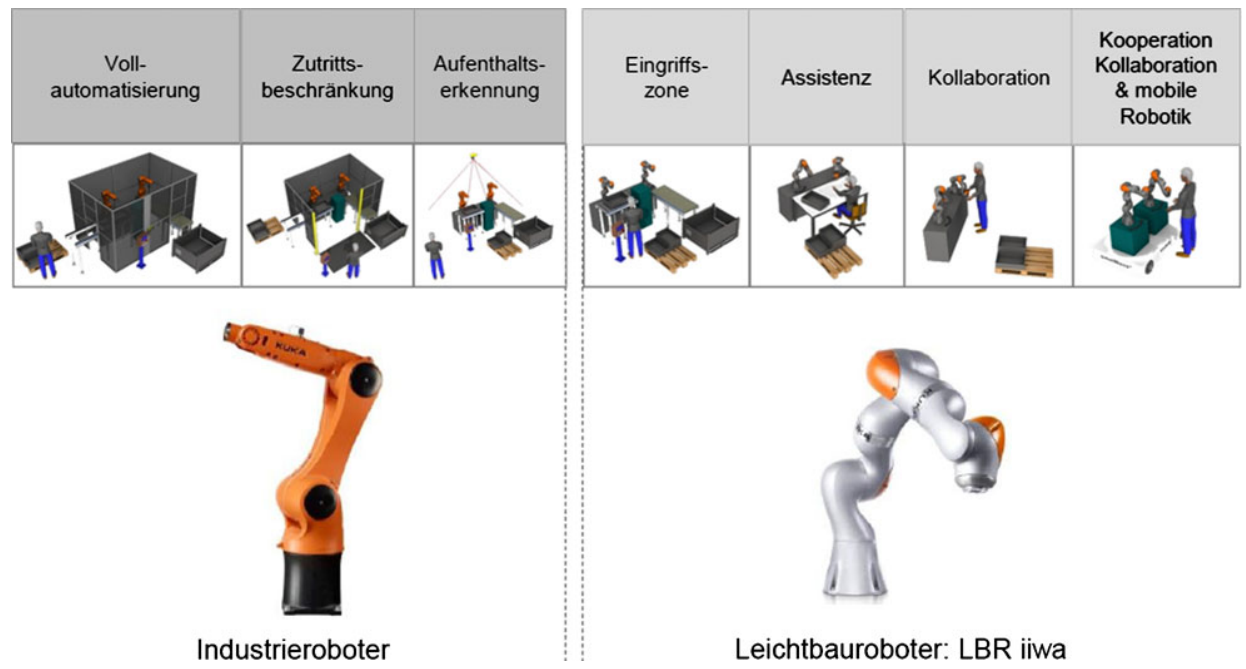

Abb. 1 Stufen der Mensch-Roboter Kooperation - von der Vollautomatisierung bis zur Kooperation. Quelle: Dr. Matthias Reichenbach: Merkmale der Mensch-Roboter-Kooperation im produktiven Umfeld, 16. IFF-Wissenschaftstage, Magdeburg 2013

tigten Ende Februar 2014 knapp 5,3 Millionen Personen tätig. Deutschland liegt mit einer Roboterdichte in der industriellen Produktion von gut 270 Robotern pro 10.000 Beschäftigte auf Platz drei hinter Japan und Korea (Schwarzkopf 2014). Es ist aufgrund neuer technologischer Entwicklungen in der Robotik zu erwarten, dass mit einer zunehmenden Flexibilisierung der Produktion die Roboterdichte weiter zunehmen wird, da der Roboter die flexible Automatisierungskomponente schlechthin ist: Roboter können verschiedenste Prozesse und Aufgaben durchführen. Was der Roboter tut, ist letztlich nur eine Frage der Programmierung und der Werkzeuge.

Damit stellt sich auch in der roboterbasierten Automation die Frage nach dem Verhältnis zwischen Mensch und Maschine. Wird die eine Hälfte der Beschäftigten Roboter „programmieren“ bzw. den Robotern zumindest Anweisungen geben und sie einlernen, während die andere Hälfte der Beschäftigten von Robotern Arbeitsanweisungen entgegennimmt und diese dann befolgt?

In der automatisierten Produktion lassen sich heute mehrere Stufen der Beziehung zwischen Mensch und Maschine unterscheiden (vgl. Abb. 1). In einer vollautomatisierten Roboterzelle ist der Roboter durch einen fest installierten Schutzzaun vom Menschen getrennt. Dieser Schutzzaun bleibt während der automatisierten Produktion geschlossen. Menschen befinden sich nicht innerhalb der Zelle. Eine wirkliche Zusammenarbeit zwischen Mensch und Roboter findet in dieser Phase nicht statt. Der Mensch kommuniziert über ein Bedienhandgerät oder eine feste Bedienstation mit der Robotersteuerung, zum Beispiel zum Auswerten von Diagnosedaten oder zur Beseitigung von Störungen. Außer während der Inbetriebnahme und Programmierung des Robotersystems und evtl. zur Beseitigung von Störungen findet keinerlei direkter Kontakt oder Zusammenarbeit zwischen Mensch und Robotersystem statt. Roboter und Mensch arbeiten jeweils autonom. 
Anstatt mit einem festen Schutzzaun, können solche Zellen auch mit einer sicheren Zutrittsbeschränkung ausgestattet sein, beispielsweise durch Trittmatten oder Lichtgitter (virtueller Schutzzaun). In diesem Fall kann der Mensch zwar die Roboterzelle betreten oder in die Zelle hineingreifen, beispielsweise um Bauteile in den Robotergreifer einzulegen. Der Roboter wird in dieser Zeit jedoch sicher stillgesetzt. Von einer tatsächlichen Interaktion zwischen Mensch und Roboter kann man auch in diesem Fall nicht sprechen.

Eine dritte Variante bilden Roboterzellen, die zwar über gar keinen Schutzzaun verfügen, deren kompletter Arbeitsbereich jedoch mit sicheren Sensoren (beispielsweise an der Decke) überwacht wird, sodass Aufenthalte von Menschen im Gefahrenbereich des Roboters sicher detektiert werden. Dringt der Mensch in den Arbeitsraum des Roboters ein, wird dessen Geschwindigkeit in Abhängigkeit vom Abstand zwischen Mensch und Roboter sicher reduziert bis hin zum Stillstand. Auch hier findet ansonsten keine weitere Interaktion zwischen Mensch und Maschine statt.

All diese Szenarien stellen den Stand der Technik dar und werden bereits heute in roboterbasierten Produktionen eingesetzt. Sie basieren auf sicheren Sensoren, welche das Eindringen des Menschen in den Arbeitsbereich des Roboters sicher erkennen und den Roboter daraufhin sicher stillsetzen (oder zumindest dessen Arbeitsgeschwindigkeit sicher reduzieren). Beispiele für tatsächliche Interaktionen zwischen Mensch und Roboter findet man hingegen nur selten im praktischen Einsatz. Bei Industrierobotern liegt dies daran, dass diese in der Regel große Massen sehr schnell bewegen können und dass die dadurch auftretende hohe Energie im Kollisionsfall mit dem Menschen nicht schnell genug abgebaut werden könnte, um diesen beim Aufprall zu schützen. So wiegt ein typischer Industrieroboter durchaus eine Tonne und das Werkzeug, welches er bewegt, zum Beispiel $150 \mathrm{~kg}$ im Falle einer Punktschweißzange. Ein Mensch darf sich nur unter ganz bestimmten Voraussetzungen im Arbeitsbereich eines solchen Roboters aufhalten. So muss die Geschwindigkeit des Roboters auf $250 \mathrm{~mm} / \mathrm{s}$ begrenzt sein (im Automatikmodus bewegt sich ein Roboter mit ca. 2 m/s) und der Mensch, der sich im Gefahrenbereich des Roboters befindet, muss während der gesamten Roboterbewegung einen Zustimmtaster betätigen. Wird dieser plötzlich losgelassen, bleibt der Roboter unverzüglich stehen. Dieser Modus wird beispielsweise während der Inbetriebnahme und Vor-Ort-Programmierung des Roboters gewählt, wenn sich der Roboterprogrammierer in der Roboterzelle aufhält. In speziellen Applikationen ist aber auch das Handführen des Roboters möglich. In diesem Fall befindet sich am Ende des Roboterarms ein sogenanntes Guiding-Device mit einem Zustimmtaster, mit dem der Bediener den Roboter in die gewünschte Richtung bewegen kann. So kann ein Roboter beispielsweise zur Handhabung großer und schwerer Bauteile verwendet werden.

Neben diesen klassischen Industrierobotern macht in letzter Zeit immer mehr eine neue Generation von Robotern von sich Reden, nämlich die der sensitiven Roboter. Sie sind kleiner und leichter als die klassischen Industrieroboter, haben weniger gefährliche Gehäusekanten, sind aber insbesondere auch mit integrierten, sicheren Sensoren ausgestattet, um gewollte oder ungewollte Berührungen mit der Außenwelt sicher detektieren zu können. Zu dieser Klasse der sensitiven Roboter zählt der neue KUKA Leichtbauroboter LBR 
Abb. 2 Sichere

Kollisionserkennung. Der KUKA LBR iiwa erkennt mit Hilfe seiner sicheren internen Gelenksensoren

Abweichungen zwischen gewünschten Kontaktkräften beim Einsetzen des Bauteils und unerwünschten Kontaktkräften bei Kollision mit der Hand des Werkers. Quelle: KUKA

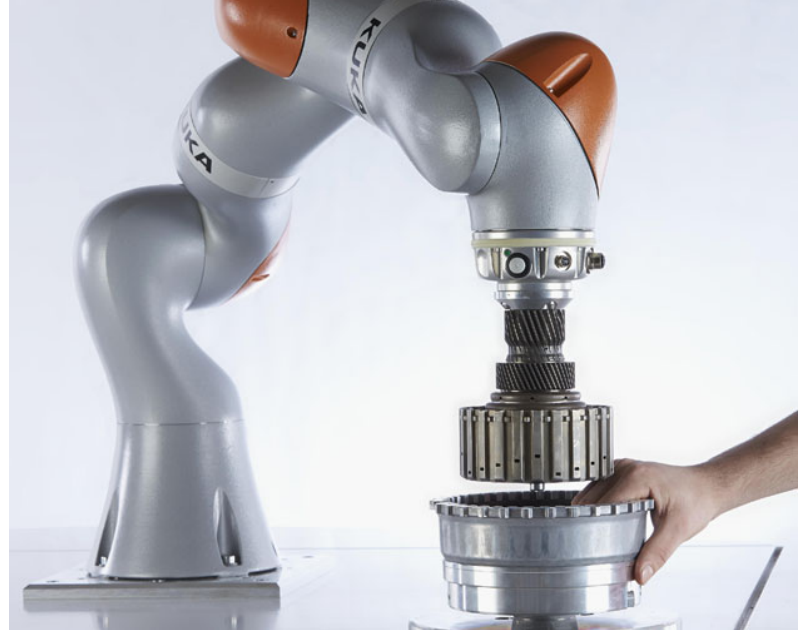

iiwa, der auf der Hannover Messe 2013 und der Automatica 2014 vorgestellt wurde, Europas größter Fachmesse für Robotik in München. Mit dem LBR iiwa sind vollkommen neue Interaktions- oder gar Kollaborationsmöglichkeiten zwischen Mensch und Roboter möglich.

Zunächst wird es damit Roboterzellen ohne Schutzzaun geben, in deren unmittelbarer Umgebung ständig Menschen arbeiten können (,Eingriffszonen“). Im Gegensatz zu den oben genannten drei Beispielen ist hier das „Eindringen“ des Menschen in den Arbeitsbereich des Roboters also nicht die Ausnahme, sondern die Regel. Auch ohne weitere sichere Sensoren in der Zellenumgebung würde der Roboter aufgrund seiner internen, sicheren Sensoren eine (unbeabsichtigte) Berührung mit dem Menschen sicher erkennen und sofort stehen bleiben (vgl. Abb. 2). Aufgrund der Außenstruktur des Roboters und seiner geringen Masse sind die Folgen einer Kollision mit dem Menschen sehr begrenzt und nicht größer, als wenn der Mensch mit einem anderen menschlichen Kollegen unbeabsichtigt zusammenstoßen würde. Eine Risikobewertung vor Inbetriebnahme der jeweiligen Anwendung ist jedoch auch in diesem Fall erforderlich. Diese wird u.a. sehr stark von dem am Roboter angebrachten Werkzeug abhängen. Ist am Roboter ein Werkzeug mit scharfen Kanten angebracht, so sind die zulässigen Geschwindigkeiten niedriger als bei einem Werkzeug mit abgerundeten Kanten.

Von solchen Roboterzellen mit Eingriffszonen, die einen unbeabsichtigten Kontakt erlauben, ist es nun nur noch ein kleiner Schritt zu Assistenzrobotern, bei denen durchaus ein Kontakt zwischen Mensch und Maschine in bestimmten Bearbeitungsschritten vorgesehen ist. Der Roboter assistiert dem Menschen beispielsweise wie eine dritte Hand, indem er dem Werker Teile anreicht, oder wie ein intelligentes Stativ, indem er sich samt Werkzeug vom Menschen in eine bestimmte Position bewegen lässt und diese dann hält. Der Mensch bewegt den Roboter dabei nicht über die Verfahrtasten eines Handbediengerätes, sondern über Gesten oder fasst ihn direkt an und bewegt ihn an die gewünschte Position (vgl. Abb. 3). 
Abb. 3 Handführen des LBR iiwa, um eine

Montage-Aufgabe einzulernen.

Quelle: KUKA

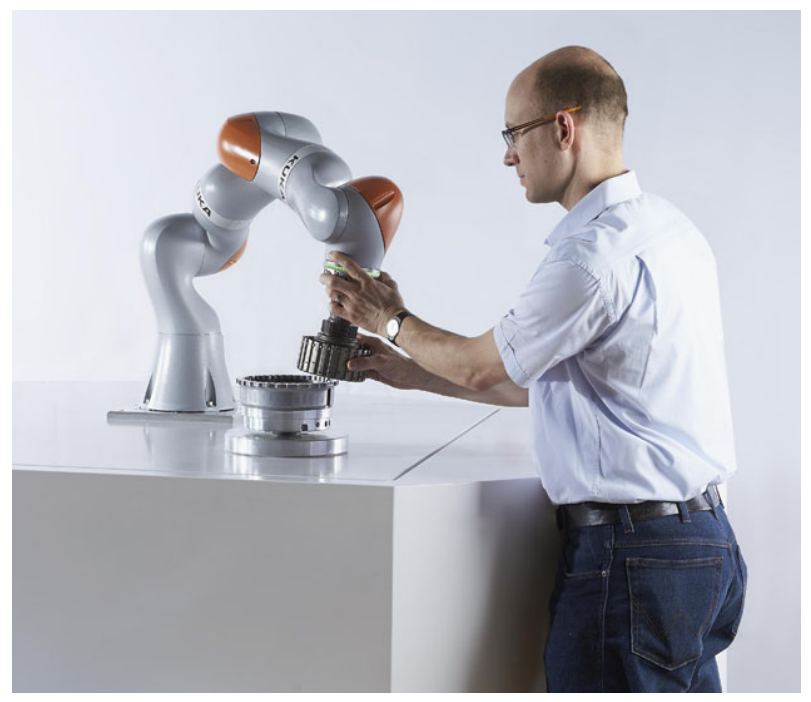

Die Kollaboration zwischen Mensch und Roboter bildet schließlich die „Königsdisziplin“. Hier teilen sich Mensch und Maschine ständig einen gemeinsamen Arbeitsbereich und können sich auch ständig berühren. Mensch und Roboter arbeiten unmittelbar an einer gemeinsamen Aufgabe. Teile der Aufgabe werden vom Menschen erledigt und andere Teile vom Roboter. So werden die komplementären Fähigkeiten von Mensch und Roboter optimal genutzt: Der Mensch hat überlegene Wahrnehmungsfähigkeiten, ist kreativ, verfügt über ein unübertroffen vielseitiges und feinfühliges „Greifsystem“ (die menschliche Hand), ist mobil und kann sich schließlich sehr schnell an neue Situationen anpassen. Der Roboter hingegen ist äußerst präzise, liefert immer eine gleichbleibend hohe Qualität, kann gefährliche Arbeiten durchführen und ermüdet auch bei sehr monotonen Tätigkeiten nicht.

In Zukunft sind noch weitere Kollaborationsformen denkbar, in denen Mensch und Maschine ,auf Augenhöhe“ zusammenarbeiten, was wiederum bedeutet, dass die Maschine neben den oben genannten integrierten Sicherheitseinrichtungen und Sensoren auch über eine gewisse Autonomie und damit über eine eigene Intelligenz verfügen muss. Der Roboter muss hierzu über ein Weltmodell verfügen (also eine stets aktuelle interne Repräsentation des Zustands seiner Umwelt) sowie über eine Wissensbasis, in der beispielsweise Informationen zu unterschiedlichen Fertigungsverfahren und Materialien hinterlegt sind. Auf Basis dieses Modellwissens muss der Roboter Schlussfolgerungen durchführen und in gewissen Grenzen auch neues Wissen erlernen können. Eine weitere Voraussetzung für die angesprochene Autonomie besteht darin, dass nicht nur der Mensch mobil ist, sondern auch der Roboter seinen Ort selbständig verändern kann.

Die dargestellten Stufen der Mensch-Roboter Kooperation lassen nicht vermuten, dass Roboter in absehbarer Zeit den Menschen Anweisungen geben werden. Bestenfalls wird durch den Roboter der Zeitpunkt eines manuellen Eingriffs festgelegt (zum Beispiel, wenn der Roboter eine Aufgabe erledigt hat und ein neues Bauteil manuell einzulegen ist, oder 
im Störungsfall). Dies ist in etwa vergleichbar mit den Anweisungen, welche der Fahrer eines Autos aus seinem Navigationssystem erhält, um sich in einer fremden Gegend zurechtzufinden. Es handelt sich hierbei also um ein Werkzeug des Menschen: Der Mensch verfolgt eine Absicht bzw. ein Ziel und bedient sich zur Zielerreichung eines geeigneten Hilfsmittels. Dass nun aber tatsächlich ein Roboter über eine eigene Problemlösekompetenz verfügt und sich der Mensch dieser Lösungsidee ,unterordnet“, ist höchstens im letztgenannten Fall denkbar, in welchem der Roboter über eine eigene Autonomie und über eine eigene Schlussfolgerungskompetenz verfügt, welche in Verbindung mit dem Zugriff auf riesige Datenmengen sowie weit verzweigte Sensornetzwerke in gewissen Grenzen als „Intelligenz“ interpretiert werden kann. Aber selbst in diesem Fall verfolgt die Maschine keine eigene Absicht. Die Ursache dafür, dass die Maschine sich so verhält, wie sie sich verhält, ist letztlich doch durch den Menschen gegeben. Entweder durch den ursprünglichen Programmierer oder durch den momentanen Auftraggeber. Eine Maschine hat von sich heraus keine Eigenmotivation, um eine Handlungsfolge zu initiieren, sondern sie erfüllt letztlich eine vom Menschen gestellte Aufgabe und bleibt damit immer ein Werkzeug des Menschen. Die These von Brynjolfsson und McAfee, dass es eine Beschäftigtengruppe geben wird, die sich von einem Automaten sagen lassen muss, was zu tun ist, ist in der Produktion also nicht in Sicht.

Open Access This chapter is distributed under the terms of the Creative Commons Attribution Noncommercial License, which permits any noncommercial use, distribution, and reproduction in any medium, provided the original author(s) and source are credited.

\section{Literaturverzeichnis}

Pearlstein, S. (2014). Review: 'The second machine age', by Erik Brynjolfsson and Andrew McAfee. The Washington Post, 17.01.2014. http://www.washingtonpost.com/opinions/review-the-secondmachine-age-by-erik-brynjolfsson-and-andrew-mcafee/2014/01/17/ace0611a-718c-11e3-8b3fb1666705ca3b_story.html.

Braunberger, G. (2014). Macht der Maschinen. FAZ, 27.04.2014. http://www.faz.net/aktuell/ wirtschaft/menschen-wirtschaft/digitale-revolution-macht-der-maschinen-12910372.html.

Schwarzkopf, P. (2014). Robotik und Automation vor großen Aufgaben. VDMA Pressemitteilung vom 19.05.2014. http://rua.vdma.org/article/-/articleview/3936490. 